\title{
Evaluación de cocinas mejoradas tipo CETA hibrida, dentro del marco del programa terrena, en comunidades vulnerables del departamento de Jinotega
}

\author{
Edwin Antonio Reyes Aguilera ${ }^{1}$ \\ Iviss Onelia Medina Benavides² \\ Lesbia Lilieth Rodríguez Tinoco ${ }^{3}$
}

\section{RESUMEN}

El objetivo principal de esta investigación es evaluar las cocinas mejoradas tipo CETA Hibrida, implementadas en comunidades de Jinotega, sus impactos en las condiciones de vida de sus usuarios y el medio ambiente. Es una investigación cuantitativa según su enfoque, con un nivel de profundidad según su tipología: exploratoria, descriptiva y correlacional. La muestra del estudio es probabilístico, 36 cocinas mejoradas y 36 familias. Los instrumentos utilizados fueron: la encuesta basada en un cuestionario estructurado, prueba controlada de cocinado para medir el rendimiento de la cocina mejorada, se instalaron equipos de medición de partículas y monóxido de carbono. Los resultados obtenidos muestran que las cocinas mejoradas contaminan menos el interior de las viviendas que las cocinas tradicionales, debido a la eficiencia y al diseño con chimenea de las cocinas mejoradas, consumen menos leña, menor tiempo en la cocción, disminuyen las enfermedades respiratorias, las cocinas mejoradas sin chimenea contaminan más que las tradicionales. Se concluye que el uso de estas cocinas es más eficiente que las tradicionales debido a su diseño y mayor concentración de calor en la cámara de combustión, menor despale de bosques y además debido a no darle seguimiento al proyecto la mayoría de estas cocinas están deterioradas.

Palabras claves: Cocinas mejoradas, monóxido de carbono, material particulado.

Recibido: 31 de agosto de 2015

Aceptado: 06 de octubre de 2015

1 UNAN-Managua, FAREM-Estelí. Correo Electrónico: edwinra11@yahoo.es

2 UNAN-Managua,FAREM-Estelí. Correo Electrónico: ivissomedina@gmail.com

3 UNAN-Managua, FAREM-Estelí. Correo Electrónico: lilieth.rodritinoco@gmail.com 


\section{Evaluation of improved stoves CETA hybrid type, part of the program TERRENA earthly program in vulnerable communities in the department of Jinotega}

\section{SUMARY}

The main objective of this research is to evaluate the improved stoves CETA Hybrid type, implemented in communities of Jinotega, its impacts on the lives of its users and the environment. It is a quantitative research, it is exploratory, descriptive and correlational. The study sample is probabilistic, 36 improved stoves and 36 families. The instruments used were: survey based on a structured questionnaire, controlled cooking test to measure the performance of the upgraded kitchen, equipment for measuring particles and carbon monoxide were installed. The results show that the improved stoves pollute less the interior of the houses than the traditional ones, due to the efficiency and design with chimney. The improved stoves consume less wood, less cooking time, decrease the respiratory diseases. The improved stoves without chimney pollute more than traditional ones. It is concluded that the use of these stoves is more efficient than traditional ones due to its design and greater concentration of heat in the combustion chamber, the less clear-cutting of forests and also due to the lack of follow up the project, most of these stoves are damaged.

Keywords: Improved cook, carbon monoxide, particulate matter. 


\section{INTRODUCCIÓN}

A consecuencia de la exposición a las emisiones producidas por la combustión de biomasa, mueren al año alrededor de 1.5 millones de personas, principalmente mujeres y niños (OMS, 2007). La combustión incompleta de la madera en fogones abiertos genera gases y partículas. En el caso de estas últimas, de la fracción respirable $\mathrm{PM}$, se registran concentraciones en el intervalo de 300 a 3,000 $\mu \mathrm{g} / \mathrm{m} 3$ en países de África, Asia y América Latina (Smith et al., 1994; McCraken y Smith, 1998; Albalak et al., 1999; Zhang et al., 1999) y pueden llegar a niveles por arriba de $10,000 \mu \mathrm{g} / \mathrm{m} 3$ (WHO, 2002), lo cual se encuentra al origen de una serie de enfermedades respiratorias.

ONGAWA Ingeniería para el Desarrollo Humano, trabaja en Nicaragua en zonas rurales desde hace más de quince años. Durante los últimos cinco años se ha ido enfocando cada vez más a la ordenación del territorio y a la gestión de los recursos naturales, tanto en el marco municipal como en el marco de la familia en lo que se han llamado Planes de Manejo Sostenible de Fincas.

Es en este marco del trabajo con las familias y las comunidades, en el que surge la actividad de la reforma de las cocinas, como un elemento necesario para disminuir el consumo de leña y mejorar el saneamiento integral de las viviendas. Hay que tener en cuenta que esta es una actividad muy concreta dentro de un Programa muy amplio, y que esta actividad aislada no podría contribuir a los objetivos que se propone el Programa Terrena (Territorio y Recursos Naturales).

Una de las líneas de acción fundamentales es la promoción y difusión de tecnologías apropiadas y modelos de gestión sostenible de los recursos naturales en el medio rural centroamericano. El programa pretende lograr un efecto multiplicador, mediante el fomento de la difusión de las experiencias exitosas del propio programa, mediante estudios y publicaciones.
Es por esto que se pretende demostrar a través de la investigación que el uso de tecnologías eficientes, como las estufas mejoradas de leña, pueden contribuir a disminuir la degradación de los recursos forestales, a mitigar las emisiones de gases de efecto invernadero y otros co-beneficios como la reducción de riesgos en la salud por la exposición a contaminantes tóxicos y la reducción del consumo de recursos y ahorro en tiempos de recolección de estos biocombustibles.

De ahí el interés por evaluar cómo las familias beneficiarias del proyecto perciben esta nueva tecnología, así como también el uso y mantenimiento que hacen de la misma, para obtener evidencias que permitan determinar los resultados del proyecto, en términos de su sostenibilidad y alineamiento a los Objetivos de Desarrollo del Milenio.

En vista de la existencia de las cocinas, es necesario hacer un estudio sobre el impacto que tienen, en áreas como el medio ambiente, salud y la economía de los beneficiarios, porque según información preliminar que se tiene, se consume menos leña en comparación con los fogones tradicionales, sin llegar a tener datos concretos sobre el verdadero impacto de los mismos en la conservación de los recursos naturales a falta de una evaluación de acuerdo a parámetros propios de evaluación energética del recurso.

\section{MATERIALES Y METODOS}

\section{Ubicación del área de estudio}

El estudio se desarrolló en el departamento de Jinotega, específicamente en las comunidades de: El Volcan y Las Trozas de Yali, San Marcos y Sabana Grande de San Rafael del Norte, Valerio y La Chichiguas en La Concordia.

Tipo de estudio: Estudio cuantitativo de carácter concluyente que se realiza mediante encuestas cara a cara y medición de variables a cocinas mejoradas. 
El universo y ámbito: 370 Cocinas Mejoradas, 370 familias evaluadas, residentes en la comunidad de Valerio, Las Chichiguas, Las Trozas, El Volcán, Sabana Grande, San Marcos.

El tipo de muestreo: Muestreo probabilístico debido a que todos los individuos tienen la misma probabilidad de ser elegidos para formar parte la muestra.

El tamaño de la muestra: 36 cocinas mejoradas y 36 familias.

\section{Fases de la investigación}

Para alcanzar los objetivos planteados, se ha utilizado un proceso metodológico dividido en tres fases:

Fase 1: Etapa de campo:

A fin de caracterizar las capacidades técnicas operativas de este tipo de cocina mejorada difundida en el departamento de Jinotega, se hizo uso de protocolos internacionales de evaluación de cocinas mejoradas. Estos protocolos fueron presentados inicialmente por voluntarios de VITA (Volunteers in Technical Assistance) y actualizados por ETHOS (Engineers in Technical and Humanitarian Opportunities of Service) y PCIA (Partnership for a Clean Indoor Air Network), y se pueden encontrar descritos detalladamente, junto con las plantillas de cálculo en formato Excel, en la página web de Aprovecho Research Center: http:// www.aprovecho.org/lab/pubs/testing.

A continuación se hará una breve descripción del protocolo y como éste se implementó durante el desarrollo de las pruebas:

\section{CCT (CONTROLLED COOKING TEST) Prueba Controlada de Cocinado}

Con la prueba de cocinado controlado se midió el rendimiento de la cocina mejorada en la preparación de un determinado alimento y se comparó con el rendimiento del método de cocción tradicional. En este caso, se seleccionó como alimento el arroz debido a que es un alimento básico en la dieta de gran parte de la población rural. Para la realización de esta prueba se hizo uso de la plantilla Excel: Controlled Cooking Test (CCT) data calculation sheet v.2.0. En este test se cocina $1 \mathrm{~kg}$ neto de arroz iniciando con el cuerpo de la cocina frío, de la misma forma que se haría tradicionalmente. Las cantidades utilizadas de cada ingrediente y el método de cocinado se repite en cada una de las pruebas y cocinas evaluadas. Los datos a obtenidos son: duración del tiempo de cocinado, la cantidad de combustible utilizado, y el peso final de los alimentos cocinados.

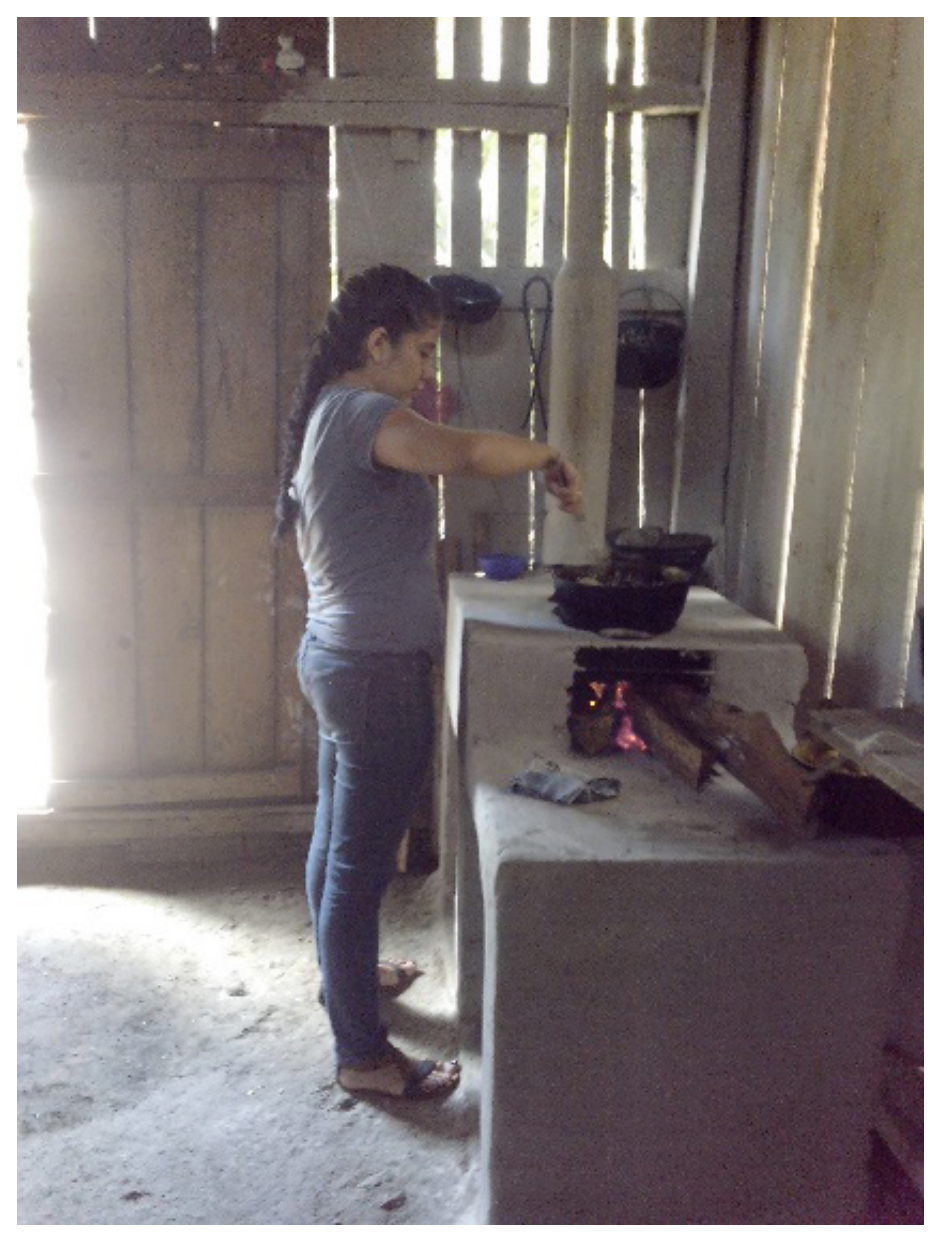

Foto 1: Prueba controlada de cocinado 
De esa manera se obtuvo el consumo específico de combustible $(\mathrm{g} / \mathrm{kg})$ que indica cuántos gramos de combustible han sido necesarios para cocinar cada kilogramo de alimento final.

\section{Mediciones de partículas (Pm2.5) y monóxido de carbono (Co)}

Durante las pruebas de CCT se llevaron a cabo mediciones continuas de emisiones, concretamente se analizaron las partículas de fracción móvil de hasta 2.5 micras de diámetro (PM 2.5) causantes de afecciones pulmonares y monóxido de carbono (CO), un gas altamente tóxico.

Durante la evolución de las pruebas, los dispositivos se colocaron a distancias normalizadas: a una altura de $140 \mathrm{~cm}$ del suelo, a una distancia lateral de 100 centímetros del borde de la cámara de combustión y separados siempre de al menos $150 \mathrm{~cm}$ de puertas y ventanas abiertas. Así mismos se realizaron estas mediciones a las 36 cocinas mejoradas y a 36 cocinas tradicionales.

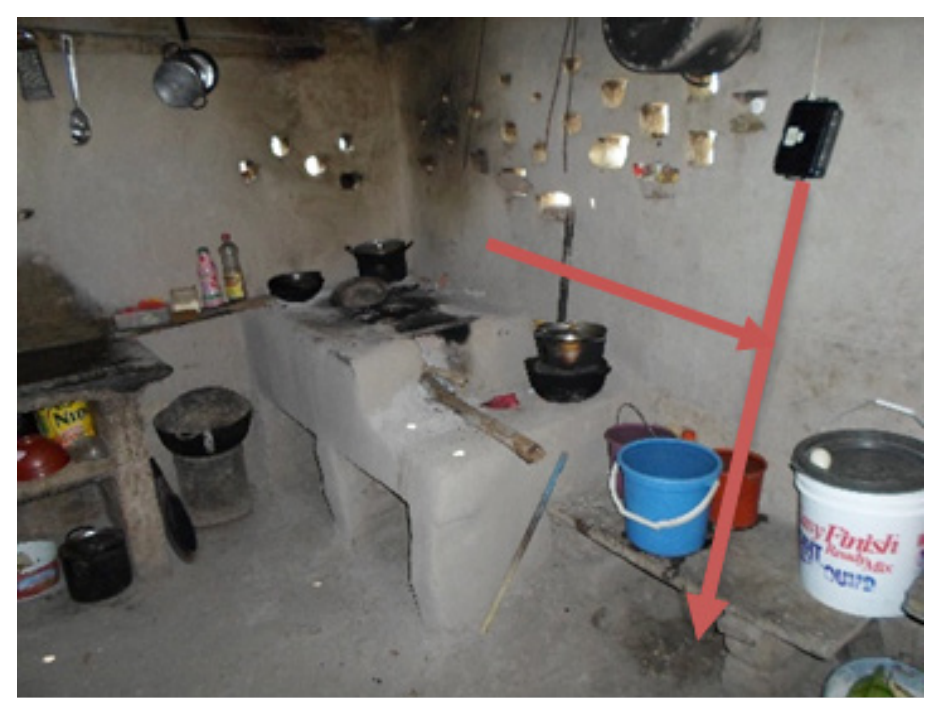

Foto 2. Colocación de equipos

La técnica utilizada para el recojo de información fue la encuesta. Ésta se basó en un cuestionario estructurado aplicado a una muestra de individuos, a quienes se les planteo una variedad de preguntas con respecto al uso de las cocinas, mantenimiento, variedad del combustible usado, problemas de salud, beneficios así como características demográficas.

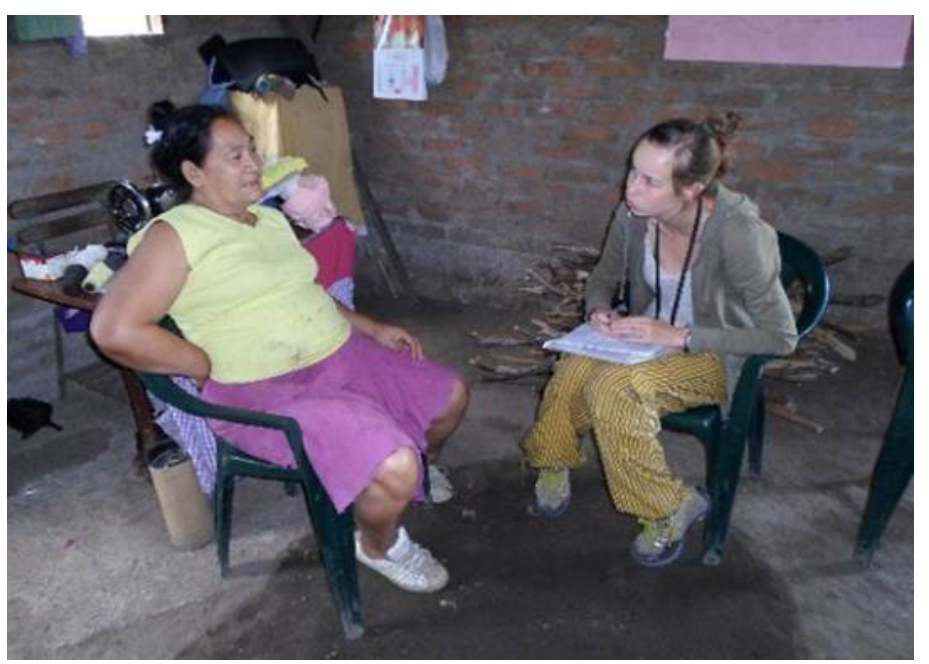

Foto 3. Aplicación de encuesta

\section{RESULTADOS Y DISCUCIÓN}

En esta sección se abordará los resultados obtenidos acerca de la evaluación realizada a las cocinas mejoradas tipo CETA Hibrida, implementadas en comunidades de Jinotega en el marco del programa TERRENA, así como sus impactos en las condiciones de vida de sus usuarios y en el medio ambiente, con el fin de identificar evidencias y lecciones aprendidas que sirvan para nuevos proyectos.

Caracterización del funcionamiento de las cocinas mejoradas a través deprotocolos internacionalmente establecidos.

\section{CCT (CONTROLLED COOKING TEST) Prueba Controlada de Cocinado}

Los datos obtenidos del CCT muestran que las cocinas mejoradas ahorran más combustible por $\mathrm{Kg}$ de alimento cocinado (grafico 1) y según la prueba el tiempo de cocinado, fue mayor en la cocina mejorada que en la cocina tradicional (grafico 2). Se observó que en la cocina tradicional la cámara de combustión es más grande por ello necesita mayor cantidad de combustible, pero por la misma razón el tiempo de 
cocinado es menor. En cuanto a tiempo de cocinado la cocina tradicional muestra gran ventaja en comparación con la cocina mejorada con casi diez minutos de diferencia, esto se debe a que las troneras y la cámara de combustión son más grandes el tiempo de finalización de cocinado fue menor y contradictoriamente utiliza más combustible.

Para comprender mejor estos resultados se muestran en los siguientes gráficos las comparaciones entre la cocina mejorada y la tradicional.

\begin{tabular}{|c|c|c|c|c|c|}
\hline Descripción & Unidades & $\begin{array}{c}\text { TEST } \\
1\end{array}$ & $\begin{array}{c}\text { TEST } \\
2 \\
\end{array}$ & Media & $\begin{array}{c}\text { Desviación } \\
\text { Estándar }\end{array}$ \\
\hline \multicolumn{6}{|c|}{ CCT (CETA Hibrida) } \\
\hline $\begin{array}{l}\text { Combustible } \\
\text { consumido }\end{array}$ & $\mathrm{gr}$ & 1734 & 1431 & 1582.50 & 151.50 \\
\hline $\begin{array}{l}\text { Equivalente de } \\
\text { combustible } \\
\text { seco } \\
\text { consumido }\end{array}$ & gr & 1237 & 1351 & 1294.00 & 57.00 \\
\hline $\begin{array}{l}\text { Peso de } \\
\text { alimentos } \\
\text { cocinados }\end{array}$ & gr & 2860 & 2936 & 2898.00 & 38.00 \\
\hline $\begin{array}{l}\text { Consumo } \\
\text { especifico de } \\
\text { combustible }\end{array}$ & $\mathrm{gr} / \mathrm{kg}$ & 432 & 477 & 454.50 & 22.50 \\
\hline $\begin{array}{l}\text { Tiempo } \\
\text { cocinado }\end{array}$ & $\min$ & 60 & 56 & 58.00 & 2.00 \\
\hline $\begin{array}{l}\text { CCT (Fogón } \\
\text { Tradicional) }\end{array}$ & Unidades & $\begin{array}{c}\text { TEST } \\
1\end{array}$ & $\begin{array}{c}\text { TEST } \\
2 \\
\end{array}$ & Media & $\begin{array}{c}\text { Desviación } \\
\text { Estándar }\end{array}$ \\
\hline $\begin{array}{l}\text { Combustible } \\
\text { consumido }\end{array}$ & $\mathrm{gr}$ & & 1281 & 1522 & 241 \\
\hline $\begin{array}{l}\text { Equivalente de } \\
\text { combustible } \\
\text { seco } \\
\text { consumido }\end{array}$ & gr & 1028 & 846 & 937 & 91 \\
\hline $\begin{array}{l}\text { Peso de } \\
\text { alimentos } \\
\text { cocinados }\end{array}$ & gr & 2662 & 2900 & 2781 & 119 \\
\hline $\begin{array}{l}\text { Consumo } \\
\text { especifico de } \\
\text { combustible }\end{array}$ & $\mathrm{gr} / \mathrm{kg}$ & 662 & 442 & 552 & 110 \\
\hline $\begin{array}{l}\text { Tiempo } \\
\text { cocinado }\end{array}$ & $\min$ & 40 & 57 & 48.5 & 8.5 \\
\hline
\end{tabular}

\section{CONSUMO ESPECIFICO DE COMBUSTIBLE}

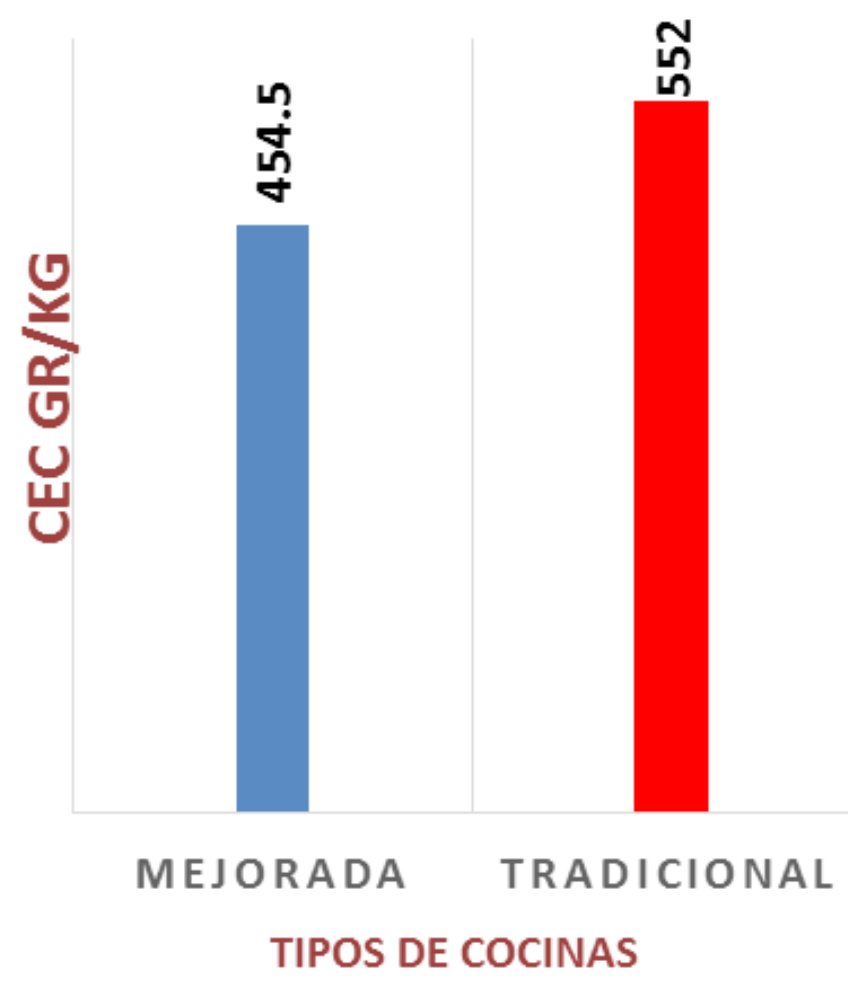

Gráfico 1. Consumo especifico de combustible
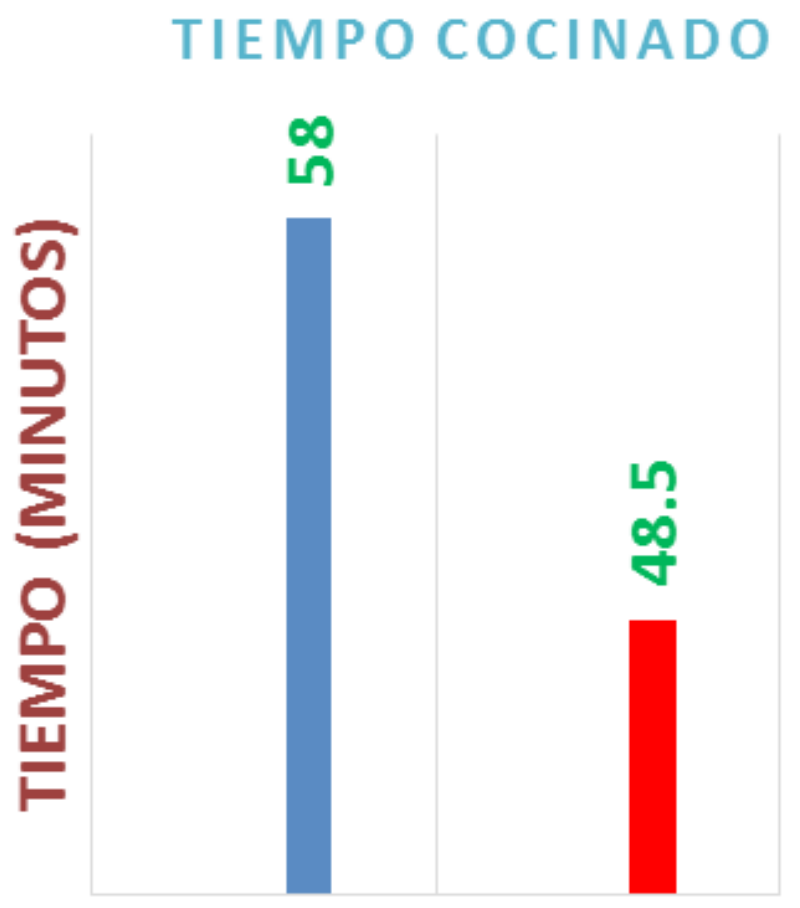

MEJORADATRADICIONAL TIPOS DE COCINA

\section{Gráfico 2. Tiempo de cocinado}


En los gráficos 3 y 4 se muestra las concentraciones de monóxido de carbono y material particulado, tomados durante los test de cocinado de las dos tipos de cocinas, la diferencia de ambas es notoria y se observa claramente que las cocinas tradicionales producen más monóxido de carbono y material particulado que las cocinas mejoradas. Esto debe a que la cocina mejorada libera los gases y material particulado al exterior de la vivienda a través de una chimenea, mientras que en la cocina tradicional las emisiones se concentran en el interior de la vivienda.

\begin{tabular}{ccccc}
\hline & \multicolumn{4}{c}{ Emisiones } \\
\cline { 2 - 5 } $\begin{array}{c}\text { CCT } \\
\text { Arroz) }\end{array}$ & \multicolumn{4}{c}{ Material Particulado PM 2.5 $(\mu \mathrm{g} / \mathbf{m 3})$} \\
\cline { 2 - 5 } & TEST 1 & TEST 2 & Media & $\begin{array}{c}\text { Desviación } \\
\text { Estándar }\end{array}$ \\
\hline $\begin{array}{l}\text { Cocina } \\
\text { mejorada }\end{array}$ & 52 & 59 & 55.5 & 3.5 \\
$\begin{array}{l}\text { Cocina } \\
\text { tradicional }\end{array}$ & 285 & 211 & 248 & 37 \\
\hline
\end{tabular}

\begin{tabular}{|c|c|c|c|c|}
\hline \multirow{3}{*}{$\begin{array}{c}\text { CCT } \\
\text { (Arroz) }\end{array}$} & \multicolumn{4}{|c|}{ Emisiones } \\
\hline & \multicolumn{4}{|c|}{ Monóxido de Carbono CO (ppm) } \\
\hline & TEST 1 & TEST 2 & Media & $\begin{array}{l}\text { Desviación } \\
\text { Estándar }\end{array}$ \\
\hline $\begin{array}{l}\text { Cocina } \\
\text { mejorada }\end{array}$ & 2.34 & 3.15 & 2.74 & 0.4 \\
\hline $\begin{array}{l}\text { Cocina } \\
\text { tradicional }\end{array}$ & 10.57 & 6.60 & 8.58 & 1.99 \\
\hline
\end{tabular}

\section{CONCENTRACION MONOXIDO DE CARBONO}

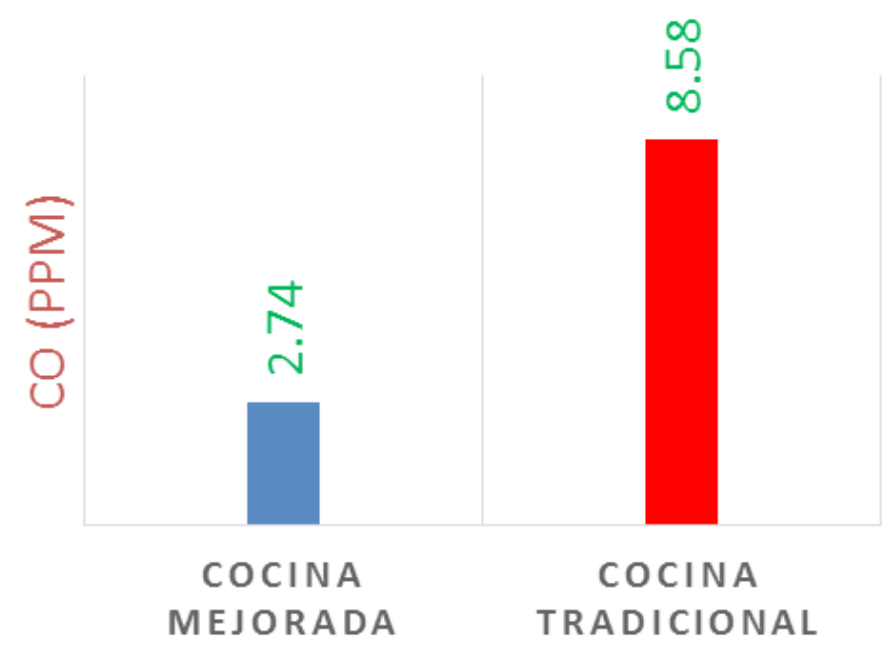

\section{TIPOS DE COCINAS \\ Gráfico 3. Concentración de CO en CCT}

MATERIAL PARTICULADO

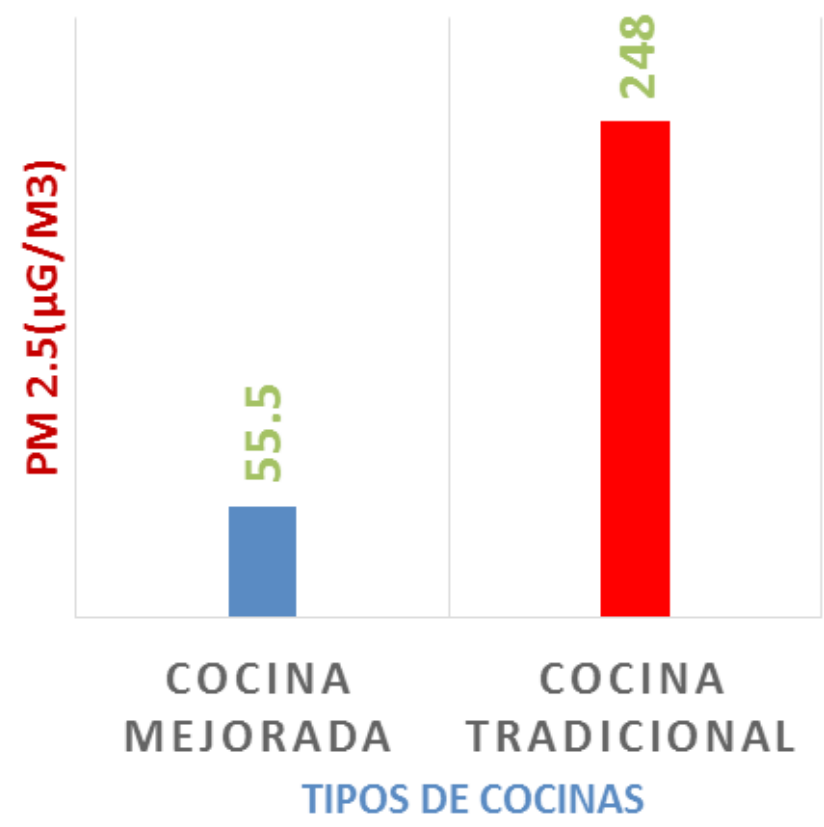

Gráfico 4. Concentración de PM en CCT 
Cuantificar las emisiones de monóxido de carbono y material Particulado que permita un análisis de la mejora en la calidad del aire interior de las viviendas de las familias receptoras de las cocinas mejoradas implementadas en el programa TERRENA.

Durante la fase de recolección de los datos a la muestra se instalaron los equipos de medición (IAP METER y el DATA USB CO) en el interior del área de cocina de las viviendas por un tiempo de 24 horas que permitió la realizaron las mediciones de concentración de monóxido de carbono y de material particulado, de la cocina mejorada y de la tradicional obteniendo los resultados que se observan en el grafico 5 y 6 .

Existe diferencias significativas en la concentración de monóxido de carbono, ya que su valor de significación es $\mathrm{P}<0.0288$; según los datos recolectados en 24 horas, las emisiones de $\mathrm{CO}$ de las cocinas tradicionales es mayor que las cocinas mejoradas, con una emisión del 58\%. Esto nos indica que la emisión de $\mathrm{CO}$ en el interior de las viviendas de las cocinas mejoradas es menor respecto a las cocinas tradicionales.

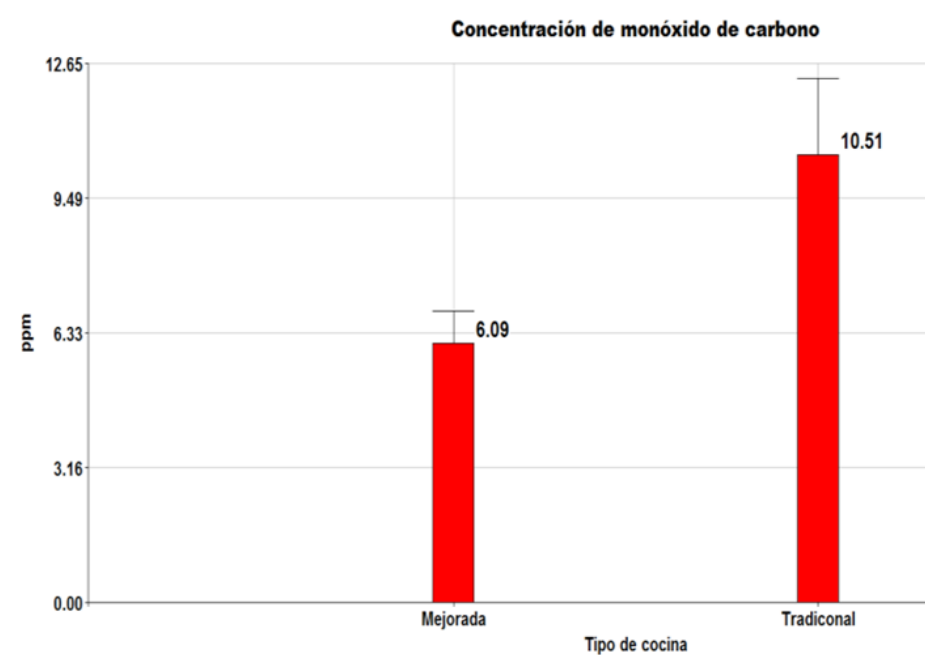

Gráfico 5. Concentración de monóxido de carbono

Es importante mencionar que en estas mediciones se tomaron tanto para cocinas mejoradas en buen estado la chimenea y cocinas mejoradas con mal estado la chimenea, lo que podríamos deducir que si se tomaran medidas solo en cocinas mejoradas con chimenea en buen estado el porcentaje de emisión de las tradicionales fuese aún mayor.

Según la guía de calidad del aire de la OMS las emisiones que produce la cocina mejorada está dentro de los rangos, es importante mencionar que en la muestra cocinas mejoradas existían algunas en mal estado, esto indica que el valor de las concentraciones sería menor si todas las cocinas estuvieran en buen estado de funcionamiento.

Existe diferencias significativas en el material particulado, ya que su valor de significación es $\mathrm{P}<0.650$; aunque se encuentre ligeramente por encima del valor $\mathrm{P}<0.500$ según los datos recolectados en 24 horas, las emisiones de MP de las cocinas tradicionales es mayor que las cocinas mejoradas, con una diferencia de 224.13 microgramos $/ \mathrm{m} 3$.

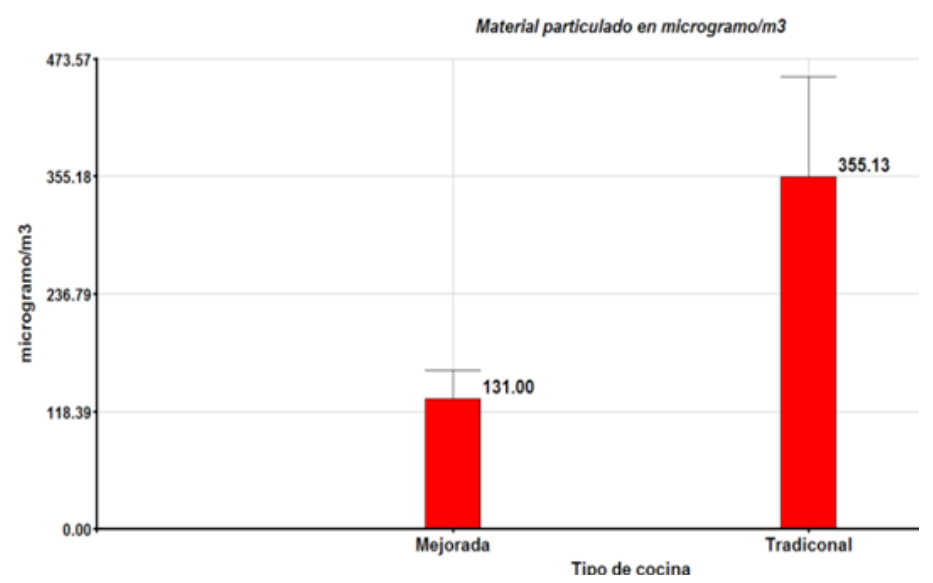

Gráfico 6. Concentración de material particulado

Cabe mencionar que para la medición de este parámetro se realizó a pocas muestras de ahí que $\mathrm{P}<0.650$, lo que nos indica que deberíamos haber hecho pruebas en un número más de muestras y la tendencia sería a por debajo de $\mathrm{P}<0.500$. Según la guía de calidad del aire de la OMS las emisiones de material particulado que produce la cocina mejorada evaluada está dentro de los rangos aun habiendo en la muestra cocinas en mal estado, esto nos indica que el valor del PM 2.5 sería menor si todas las cocinas estuvieran en buen estado. 
Con la finalidad de realizar comparaciones entre cocinas mejoradas en buen estado y cocinas mejoradas en mal estado se puede observar claramente un aumento en la emisión de monóxido de carbono de las cocinas en mal estado mayor a las que están en buen estado, debido a que el humo siempre queda en el interior del espacio donde está ubicada la cocina, y esto sumado en muchos casos a la mala ventilación.

Cabe mencionar que para la medición de este parámetro se realizó a pocas muestras de ahí que $\mathrm{P}<0.650$, lo que nos indica que deberíamos haber hecho pruebas en un número más de muestras y la tendencia sería a por debajo de $\mathrm{P}<0.500$. Según la guía de calidad del aire de la OMS las emisiones de material particulado que produce la cocina mejorada evaluada está dentro de los rangos aun habiendo en la muestra cocinas en mal estado, esto nos indica que el valor del PM 2.5 sería menor si todas las cocinas estuvieran en buen estado.

Con la finalidad de realizar comparaciones entre cocinas mejoradas en buen estado y cocinas mejoradas en mal estado se puede observar claramente un aumento en la emisión de monóxido de carbono de las cocinas en mal estado mayor a las que están en buen estado, debido a que el humo siempre queda en el interior del espacio donde está ubicada la cocina, y esto sumado en muchos casos a la mala ventilación.

En el grafico 7 se muestran diferencias de cerca de 6 ppm de $\mathrm{CO}$ de las cocinas mejoradas sin chimenea o chimenea en mal estado y con chimenea. El principal problema ha sido la falta de mantenimiento a las chimeneas y por esta razón sufrieron los daños que tienen, por el contrario las personas que le brindan mantenimiento a sus chimeneas, las cocinas funcionan perfectamente y los usuarios que tuvieron inconveniente con estas optaron por no repáralas y seguirlas utilizando.

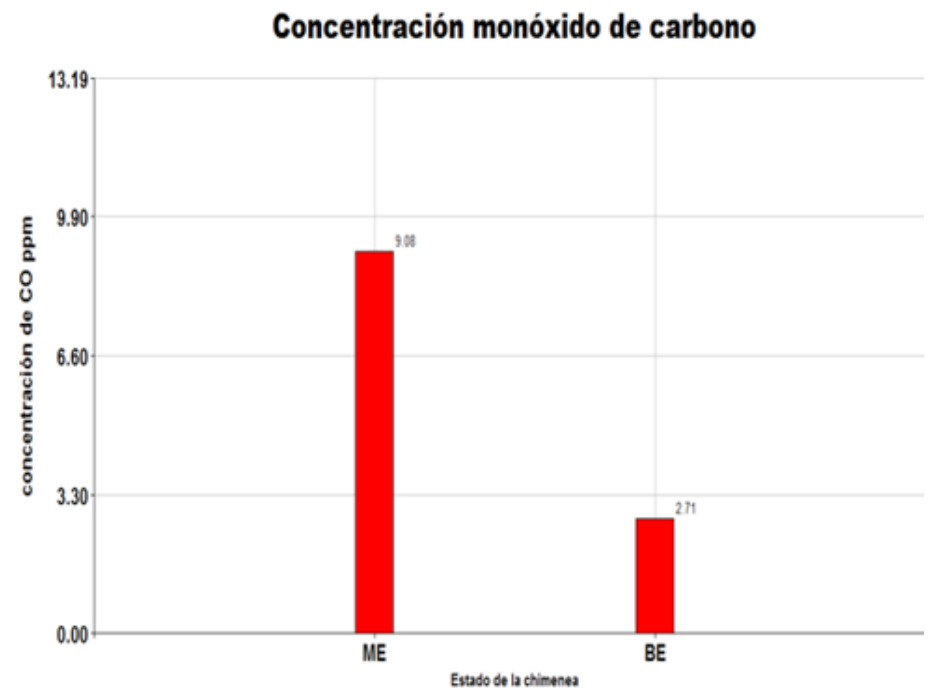

Gráfico 7. Concentración de monóxido de carbono

Evaluar la percepción de las familias usuarias de las cocinas mejoradas en cuanto a las mejoras en las condiciones de vida producidas debido al uso de cocinas mejoradas

En este acápite se abordan los resultados obtenidos a través de la aplicación de la encuesta, acerca de la percepción de las familias beneficiadas que usan la cocina mejorada.

\section{Uso de las cocinas mejoradas}

El 100\% de las familias beneficiarias tiene en uso las cocinas mejoradas casi siempre, cabe mencionar que algunas han sido modificadas, sobre todo cuando la trasladan de un lugar a otro.

\section{USOS DE LA COCINA}

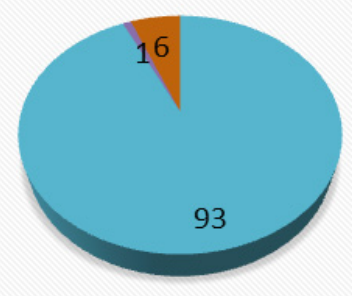

- Tipos de uso de la cocina a Doméstico, - NEGOCIO

DOMESTICO/NEGOCIO 


\section{Tipos de uso de la cocina}

El 93\% es para uso doméstico, el 1\% para negocio y el $6 \%$ para negocio y doméstico.

\section{Persona que recoge la leña}

Según las encuestadas el $88 \%$ lo recoge el hombre, el $6 \%$ las mujeres, el $2 \%$ niños y el $4 \%$ compra la leña. Este resultado demuestra que en la mayoría de las familias los hombres se encargan de la recolección de la leña, porque son ellos mismos los que salen de la casa a trabajar en el campo y de esta manera realizan las dos actividades.

El 6\% de mujeres que se encargan de la recolección de la leña son mujeres solteras o que en su hogar no hay un hombre que realice esta actividad, de igual manera el $2 \%$ de los niños que en su mayoría son hijos de madres solteras.

\section{PERSONA QUE RECOGE LA LEÑA}

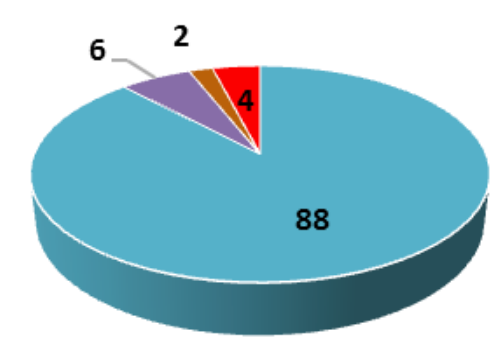

- Persona que recoge la leña - HOMBRE

- mujeres

- MUJERESY NIÑOS

- COMPRA

\section{Tiempo de recolección de la leña}

El $90 \%$ de los encuestados asegura tardarse más en recolectar la leña puesto que esta se encuentra más distante a sus hogares, el 6\% dice que no, debido a que son familias que tienen gran cantidad de terreno y además usan los desechos de la poda forestal de sus fincas, y un $4 \%$ la compra.
La mayoría de los encuestados aseguro que el avance de la frontera agrícola, la perdida de las especies forestales, la deforestación y otros aspectos inciden en el tiempo de recolección de hace unos años hasta hoy, muchas personas afirmaron que antes les era más fácil la recolección porque había más especies forestales, más vegetación y menos población.

E1 4\% de la población que compra la leña es porque no se dedica a actividades agrícolas y además se encontró que estas familias viven en una mejor situación económica lo que les facilita la compra de leña, pero igualmente aseguran que a medida que pasa el tiempo el costo de esta aumenta por las mismas situaciones antes mencionadas.
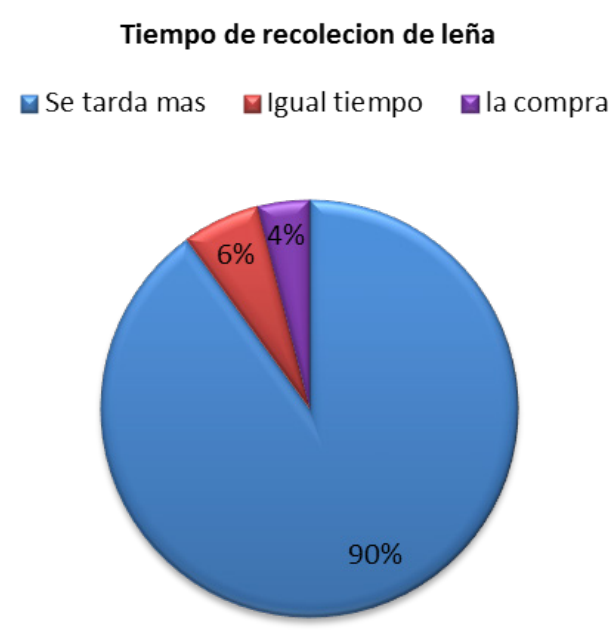

\section{Forma de recolecta de la Leña}

E1 95\% recoge la leña es decir la cortan y la llevan a sus hogares, un $4 \%$ la compra a personas que se dedican al corte y venta de leña y el $1 \%$ la recoge y la compra.

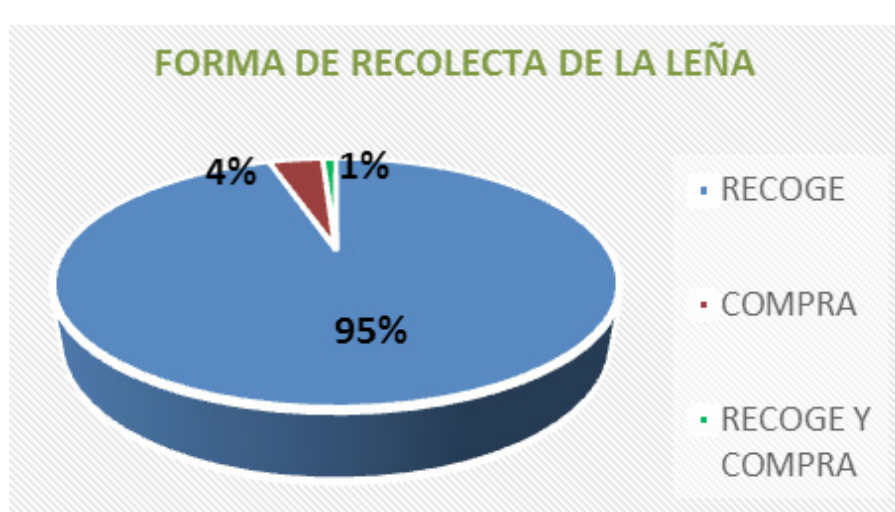




\section{Cantidad de leña utilizada diaria}

Según pesaje realizado a la cantidad de leña que las familias utilizan diariamente tenemos que: las cocinas mejoradas consumen 9,6 kilogramos/día de leña y las cocinas tradicionales consumen 12.8 kilogramos/día de leña, lo que nos demuestra que las tradicionales consumen más leña que las mejoradas, por lo tanto el despale es mayor para el uso de estas.

\section{Cantidad de leña utilizada}

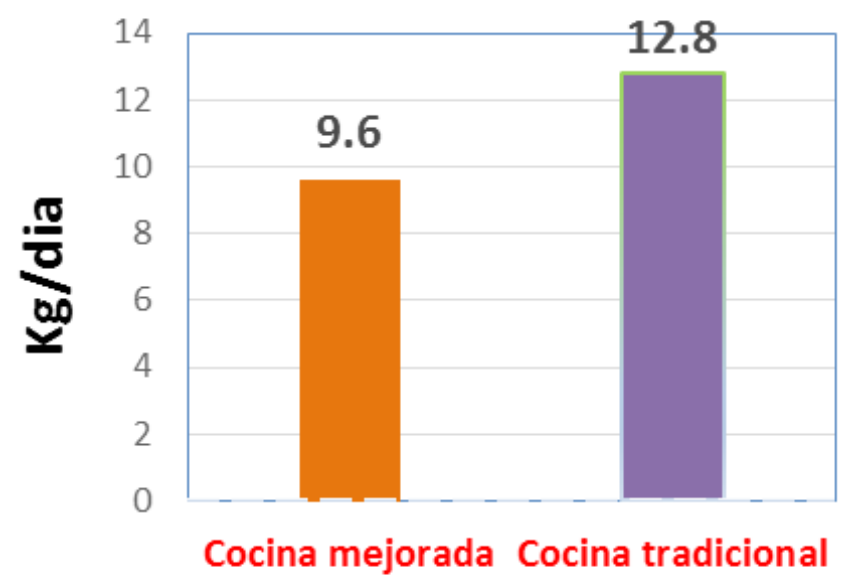

Tipos de cocinas

Esto se debe a que las cocinas mejoradas tienen un diseño que optimiza el uso del combustible y cuenta con una válvula en la chimenea que hace más fácil el control de la combustión reduciendo las perdidas térmicas en la cámara de combustión, ninguna de las familias usa la cocina correctamente, ninguna usaba la compuerta lo que nos indica que si hicieran uso eficiente de la cocina la cantidad de leña utilizada sería menor.

\section{Disminución del humo en el interior de las viviendas}

Según respuestas de las encuestadas un 52\% asegura que existe disminución de humo dentro de sus viviendas con respecto al uso de cocinas tradicionales, en cambio un $48 \%$ dice no hay disminución de humo. Obviamente dentro de estas que aseguran no haber disminución se encuentran las que tienen cocinas mejoradas en mal estado.

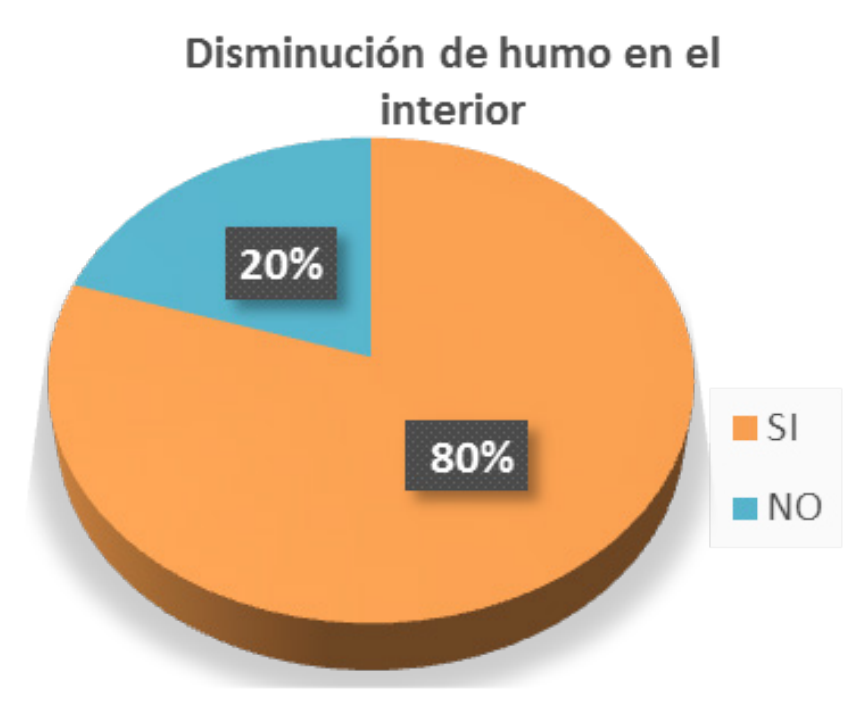

\section{Disminución de problemas visuales producidos por el humo}

De acuerdo a las encuestadas el 55\% dice haber menos problemas visuales y el $45 \%$ asegura no existir disminución de estos problemas. Obviamente dentro de estas que aseguran no haber disminución se encuentran las que tienen cocinas mejoradas en mal estado.

\section{Disminución de problemas visuales}

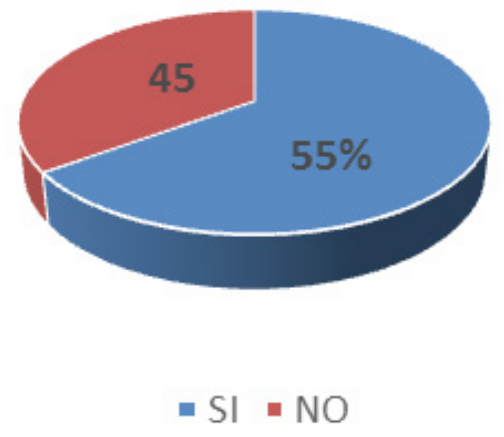

\section{Disminución de problemas respiratorios}

Un $67 \%$ de las personas manifiestan no haber disminución de los problemas respiratorios, en cambio el 33\% asegura haber reducido estos problemas con el uso de la cocina mejorada. En este 33\% se encuentran las personas que tienen dañada la chimenea de sus cocinas o usan combustible con un alto contenido de humedad. 


\section{Disminución de los problemas de salud en niños}

El 58\% de encuestados dice no haber disminución en los problemas de salud de niños asociados al CO y MP emitidos por estas cocinas y el $42 \%$ dice que los niños se enferman menos desde que adoptaron estas cocinas mejoradas.

\section{Disminución de problemas de salud en niños}

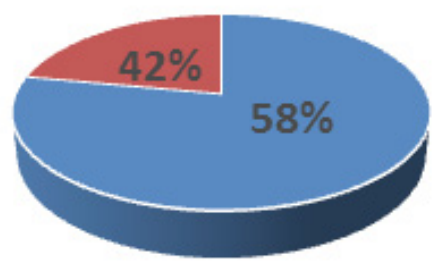

$$
\text { - } \mathrm{SI} \cdot \mathrm{NO}
$$

\section{Disposición a pagar por una cocina mejorada}

Cabe mencionar que las cocinas fueron donadas, al realizar esta pregunta en caso que la cocina actual se les dañara, el $67 \%$ responde que no está dispuesto a pagar, por factores como bajos recursos económicos, el 33\% asegura pagar porque cree han mejorado su situación de salud, estética del hogar y porque contribuye al medio ambiente.

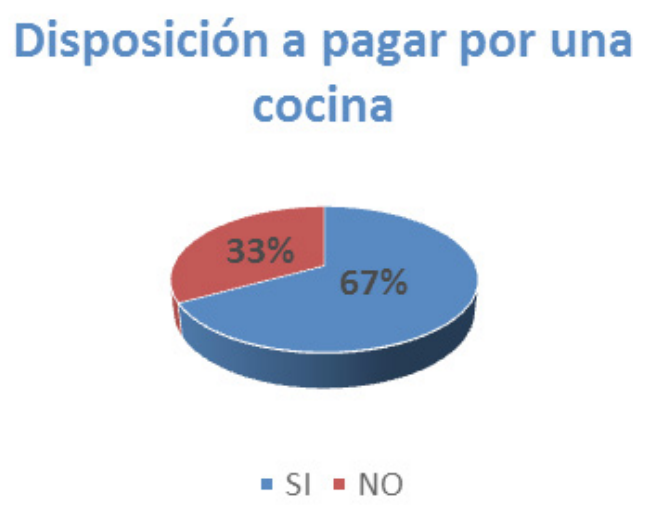

Un factor que influye en la negativa de pago es que la mayoría de la población forma parte de proyectos donde no hay una responsabilidad compartida entre el beneficiario y el proveedor y por esto se les hace fácil no darles el mantenimiento para alargar la vida útil de la cocina.

\section{Grado de satisfacción hacia la cocina mejorada}

En relación a esta pregunta las encuestadas manifiestan con un $80 \%$ estar altamente satisfechas con estas cocinas, un $15 \%$ se encuentra medias satisfecha y el $5 \%$ menciona un grado de satisfacción bajo con el uso de estas cocinas. Estos resultados se deben a que no se hace un uso adecuado de la cocina por que la capacitación del uso y mantenimiento de la cocina fue dirigido al sexo masculino y deducimos que la información no fue trasmitida correctamente a las usuarias de las cocinas. Las usuarias no tuvieron un acompañamiento constante y exigente del organismo responsable que las motivara a cuidar y darles mantenimiento a las cocinas, por ello la insatisfacción de estas.

\section{Grado de satisfacción con la cocina mejorada}

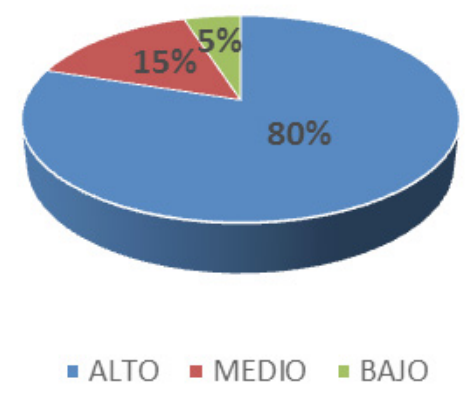

\section{CONCLUSIONES}

- Las cocinas mejoradas consumen menos leña que las tradicionales por alimento cocinado, esto permite que se despale menos árboles en la zona de incidencia del proyecto.

- Las cocinas tradicionales emiten más monóxido de carbono y material Particulado que las cocinas mejoradas, por lo cual existe mayor riesgo de enfermedades respiratorias, visuales y cardiopatías, en mujeres y niños que son los que permanecen más tiempo en el interior del área de cocinado.

- Ha existido bastante aceptación al uso de las cocinas mejoradas tipo CETA Hibrida por parte de la población beneficiaria y prueba de ello es que todas están en uso. 
- La mayoría de las cocinas mejoradas en mal estado se debe a que no se le da el mantenimiento requerido y cuando se les daña la chimenea esta no es reparada.

- Las personas encargadas de recolectar la leña tardan más tiempo en recoger la leña porque ahora se encuentra más distante debido al despale de las zonas.

- Con el uso de las cocinas mejoradas se mejora la calidad del aire interior en estas viviendas por lo tanto se reducen los riegos de contraer enfermedades.

- No existe un seguimiento por parte de los organismos financieros y ejecutores que permita monitorear el buen funcionamiento y uso de estas cocinas mejoradas.

\section{AGRADECIMIENTOS}

Este estudio se llevó a cabo gracias al financiamiento de la Universidad Politécnica de Madrid (UPM) de España, de manera especial a la doctorante Candela de la Sota por su asesoría y ayuda técnica, se agradece así mismo a la Facultad Regional Multidisciplinaria de Estelí por el apoyo económico a uno de los investigadores.

\section{BIBLIOGRAFÍA}

Córdova, U., \& Castro, A. (2012). Genero y cocinas mejoradas. Lima.

González Expósito, J. O. (2013). Estudio comparativo de cocinas mejoradas en Nicaragua. Valencia.

Korc, M., \& Quiñones, M. (2003). Diagnostico comparativo de la calidad del aire de los interiores de las viviendas de dos poblaciones indigenas del Peru. Lima.

Morales, I. M., Blanco Acevedo, V., \& García Nieto, A. (2010). Calidad del aire interior en edificios de uso público (primera ed.).

Pilco Mamani, V. (2009). Proyecto de reglamento para la evaluación y certificación cocinas mejoradas. Lima. 